\title{
Anatomical Changes in the Pelvis after Modified Chiari Pelvic Osteotomy
}

\author{
MANABU KUBO \\ Department of Orthopaedic Surgery, Kurume University School of Medicine, \\ Kurume 830-0011, Japan
}

\begin{abstract}
Summary: Although plain X-ray analysis is able to reveal anatomical changes in the frontal plane of the pelvis after Chiari pelvic osteotomy involving medial displacement (medialization) in the distal fragment and lateral displacement (lateralization) in the proximal fragment, changes in the horizontal or sagittal plane can not be discerned. Here, I have investigated three dimensional changes in the pelvis using CT in 22 patients before and after Chiari pelvic osteotomy. The various changes were investigated. Medialization in the distal fragment (average $0.4 \mathrm{~cm}$ ) occurred in the 14 cases and to a slight extent in the other 8 cases. Also anterior or posterior displacement, and anterior rotation occurred in the distal fragment. Lateralization in the proximal fragment (average $0.8 \mathrm{~cm}$ ) occurred in 19 cases and to a slight extent in the other 3 cases. Furthermore, the acetabular coverage over the femoral head, assessed by superimposing the acetabular region over the femoral head, improved by about $30 \%$ of the anterior half of femoral head and by about $20 \%$ of the posterior half of femoral head. It is suggested that the acetabular coverage over the femoral head is most affected by lateralization in the proximal fragment.
\end{abstract}

Key words CT, dysplasia, Chiari pelvic osteotomy

\section{INTRODUCTION}

In 1953, Chiari [1] first presented his pelvic osteotomy. In his original osteotomy technique, the patient is placed in the supine position and pelvic osteotomy is performed through the Smith-Petersen's anterior approach using a Gigli saw. In 1974, Chiari reported a biomechanical efficacy obtained by medialization in the distal fragment with respect to reduction in stress in the hip, and named this surgery "medial displacement osteotomy of the pelvis" [2]. Since then, further studies on where and how to displace the bone fragments after surgery have been reported. Küsswetter et al. [3] reported medialization in the distal fragment as a tilting movement around an axis running through the middle of the symphysis from ventral to dorsal. Lateralization in the proximal fragment around the axis of the sacroiliac joint (door effect) has been demonstrated using plain X-ray [4], computed tomography (CT) [5], and three-dimen- sional computed tomography (3DCT) [6].

We have performed [7-10] this operation through the lateral approach modified with detachment of the greater trochanter. The advantages of this modified method are as follows: dome osteotomy is possible along the superior acetabulum using a Tuke bonesaw, simultaneous osteotomy of the femur can be easily performed, and the greater trochanter can be fixed at an optimal position. Nonetheless, how bone fragments become displaced in three-dimensions following surgery, how simultaneous osteotomy of the femur affects the displacements of bone fragments, and how the load area increases are all uncertain. These questions were addressed in the present study using CT.

\section{PATIENTS AND METHODS}

Twenty-two hip joints of 22 dysplasia patients were examined. The patients consisted of 21 women 


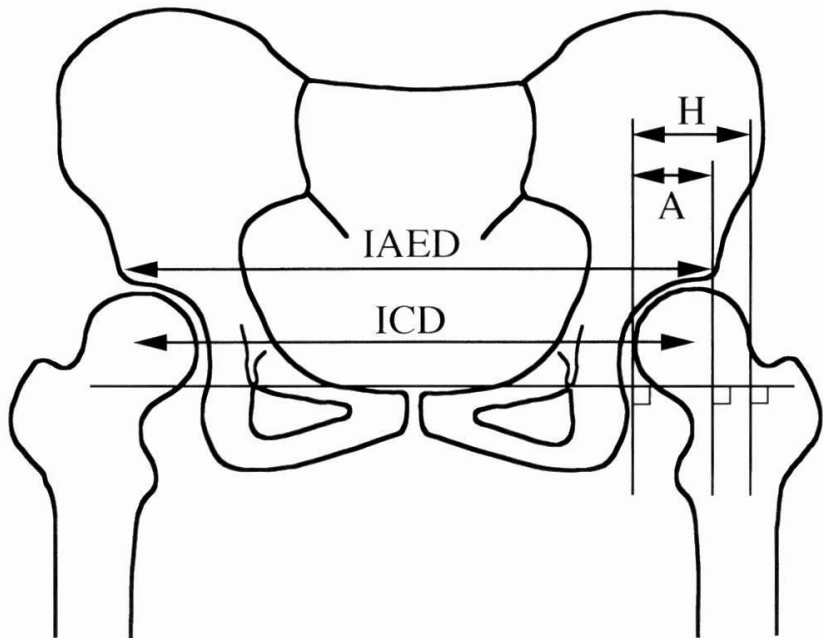

Fig. 1. Illustrated parameters were measured on A-P view on a plain radiogram. AHI: acetabular head index $=\mathrm{A} / \mathrm{H} \times 100$; ICD: inter-capital distance; IAED: inter-acetabular edges distance

a
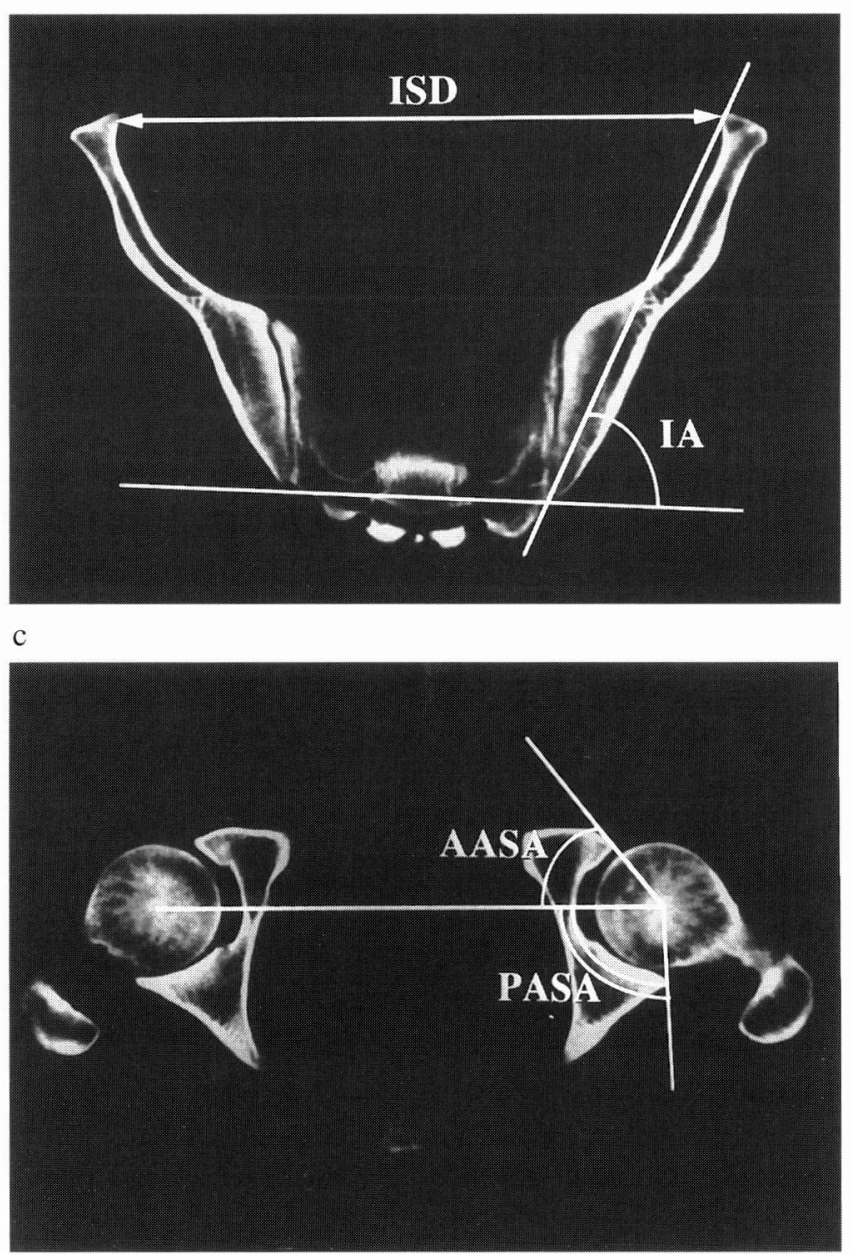

and 1 man, and their age ranged from 21 to 57 years with an average of 42.3 years. Simple Chiari pelvic osteotomy was performed on 8 patients, and Chiari pelvic osteotomy with simultaneous valgus osteotomy of the femur was performed on the other 14 patients. The indications for combined femoral valgus osteotomy were needing: improvement in congruency, having a deformity in the femoral head, and an advanced stage of coxarthrosis. Each of these operations constituted a first-time surgical procedure for the hip. Anatomical changes were investigated using anterior and posterior X-ray and pelvic CT that were taken at several days before surgery and at three months after surgery.

Changes on the plain A-P radiographs between before and after the surgery were evaluated by measurements of the Acetabular Head Index (AHI) [11], the Inter-Capital Distance (ICD), and the Inter-

b

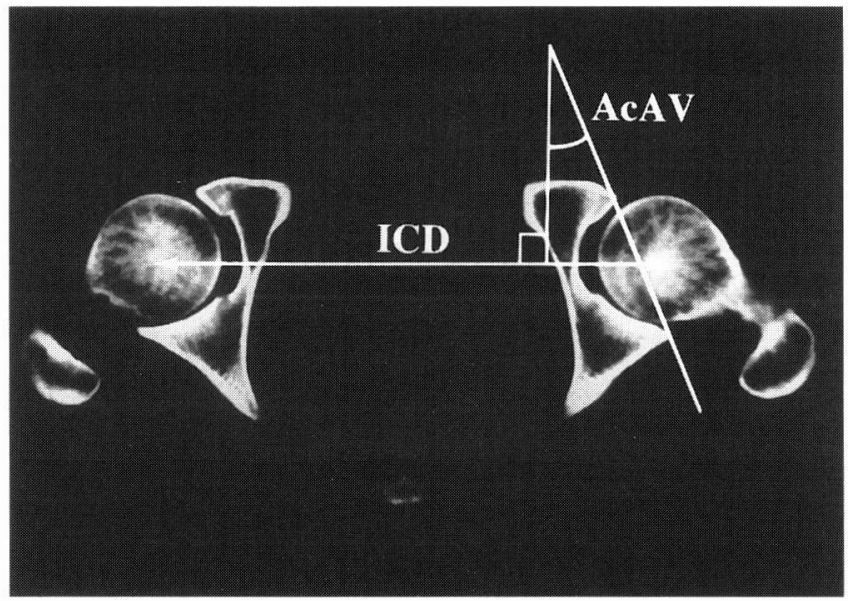

d

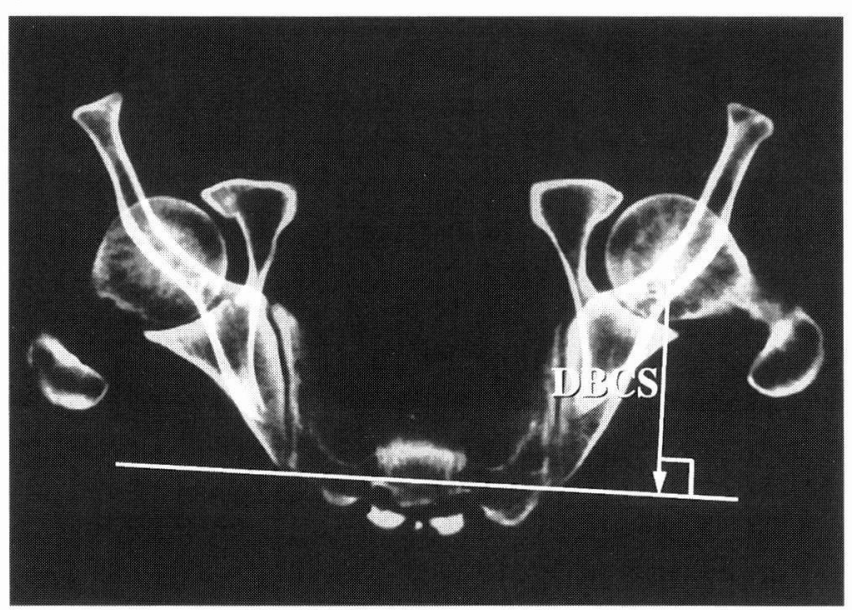

Fig. 2. Illustrated parameters were measured on a computed tomogram. a: level at the antero-superior iliac spine, ISD: inter-spinous distance; IA: iliac angle. b: level at the center of the femoral head, ICD: inter-capital distance; AcAV: acetabular anteversion. c: level at the center of the femoral head, AASA: anterior acetabular sector angle; PASA: posterior acetabular sector angle. d: superimposed CT between the antero-superior iliac spine and the center of the femoral head, DBCS: distance between caput and sacroiliac joints 
Acetabular Edges Distance (IAED) (Fig. 1). Pelvic CTs were taken at the levels of the anterior superior iliac spine, the superior acetabulum, and through the center of the femoral head, and each computer tomogram was read at a resolution of 200 dpi into a personal computer (Macintosh 8100/120). The following parameters were measured using MiniCad 5.0J software. At the level of the anterior superior iliac spine, two parameters showing the displacement of the proximal fragment were measured: the InterSpinous Distance (ISD: the distance between bilateral anterior superior iliac spine), and the Iliac Angle (IA: the angle made by the line connecting the bilateral posterior sacroiliac joint and the iliac wing) (Fig. 2a). At the level of the center of the femoral head, the Inter-Capital Distance (ICD) [5] that indicated medialization of the distal fragment, the Acetabular Anteversion (AcAV: the angle made by the line connecting the anterior and posterior acetabulum and the line perpendicular to the line connecting the bilateral femoral heads), the Anterior Acetabular Sector Angle (AASA: the angle made by

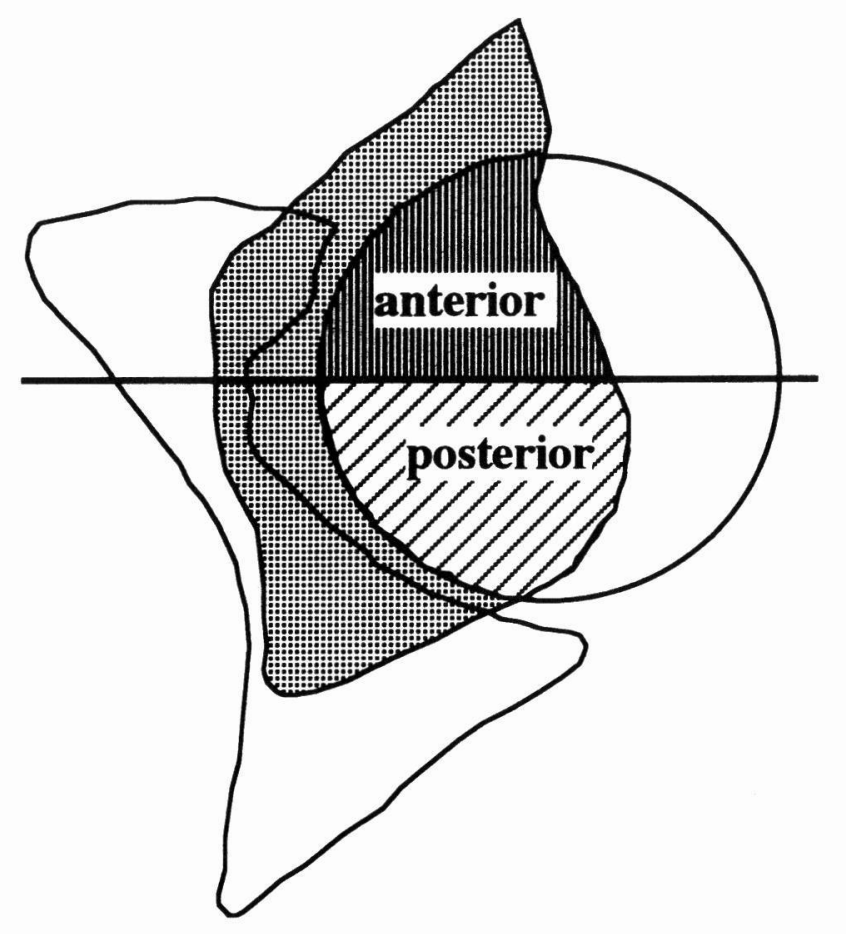

Fig. 3. The slice at the level of center of the femoral head was superimposed with that at the level of the superior acetabulum. The acetabular coverage over the femoral head was divided into anterior and posterior parts in order to evaluate the degree of improvement after surgery. This figure showed left dysplasic hip for preoperation. the line connecting the center of the femoral head and the anterior acetabulum and the line connecting the bilateral femoral heads), and the Posterior Acetabular Sector Angle (PASA: the angle made by the line connecting the center of the femoral head and the posterior acetabulum and the line connecting the bilateral femoral heads) were each measured [12] (Figs 2b, c).

Although displacement in the frontal and horizontal planes of the pelvis can be evaluated using plain X-ray and CT analysis, movement in the sagittal plane is difficult to measure using either of these methods. In order to evaluate any anterior or posterior rotation in the sagittal plane, I have measured the AcAV and the sector angle (AASA and PASA) reliability of which had already been confirmed by Anda et al. [13]. Furthermore, in order to evaluate the anterior-posterior displacement, I measured the Distance Between the Caput and Sacroiliac joints (DBCS: the distance between the center of the femoral head and the line connecting the bilateral sacroiliac joints by superimposing CT between the anterior superior iliac spine and the center of the femoral head) (Fig. 2d).

The acetabular coverage over the femoral head was assessed by superimposing CT between the acetabulum and the femoral head. Then, using NIH image 1.58 software, the area of the acetabular coverage over the femoral head was calculated. The degree of improvement in the acetabular coverage over the femoral head was evaluated in the anterior half and in the posterior half [5,14] (Fig. 3).

\section{RESULTS}

Comparison of plain A-P radiographs taken before and after surgery revealed the coverage over the femoral head (AHI), materialization in the proximal fragment (IAED), and medialization in the distal fragment (ICD) (Table 1). Changes in the ICD,

TABLE 1.

$X$-P parameters were measured before and after operation $($ mean $\pm S D)$

\begin{tabular}{ccc}
\hline X-P parameter & Preop. $(\mathrm{n}=22)$ & Postop. $(\mathrm{n}=22)$ \\
\hline AHI $(\%)$ & $56.2 \pm 7.4$ & $84.2 \pm 9.7^{*}$ \\
ICD $(\mathrm{cm})$ & $20.6 \pm 1.5$ & $20.2 \pm 1.6^{*}$ \\
IAED $(\mathrm{cm})$ & $21.8 \pm 1.4$ & $22.6 \pm 1.5^{*}$ \\
\hline
\end{tabular}

Student's t test for paired data was used to determine statistical significance. Significant difference from preoperation; ${ }^{*} \mathrm{p}<0.0001$ 
TABLE 2.

$C T$ parameters were measured before and after surgery (mean $\pm S D)$

\begin{tabular}{lllll}
\hline & \multicolumn{1}{c}{ CT levels } & CT parameters & Preop. $(\mathrm{n}=22)$ & Postop. $(\mathrm{n}=22)$ \\
\hline $\begin{array}{l}\text { Proximal } \\
\text { fragment }\end{array}$ & Antero-superior & ISD $(\mathrm{cm})$ & $20.7 \pm 2.1$ & $21.5 \pm 2.0^{* *}$ \\
& iliac spine & IA $\left(^{\circ}\right)$ & $62.9 \pm 4.9$ & $60.2 \pm 5.5^{* *}$ \\
\hline \multirow{2}{*}{$\begin{array}{l}\text { Distal } \\
\text { fragment }\end{array}$} & Center of the & ICD $(\mathrm{cm})$ & $18.4 \pm 1.2$ & $18.0 \pm 1.3^{* *}$ \\
& femoral head & AASA $\left(^{\circ}\right)$ & $15.5 \pm 7.1$ & $13.9 \pm 7.9^{*}$ \\
& & PASA $\left(^{\circ}\right)$ & $52.6 \pm 13.5$ & $53.4 \pm 14.1$ \\
& & DBCS $(\mathrm{cm})$ & $84.8 \pm 9.7$ & $82.1 \pm 8.5^{*}$ \\
\hline
\end{tabular}

Student's t test for paired data was used to determine statistical significance.

Significantly different from preoperation; ${ }^{*} \mathrm{p}<0.05,{ }^{* *} \mathrm{p}<0.0001$

TABLE 3.

The acetabular coverage over the femoral head was measured before and after surgery (mean $\pm S D)$

\begin{tabular}{|c|c|c|c|}
\hline & Preop. $(n=22)$ & Postop. $(n=22)$ & control $(n=10)$ \\
\hline Anterior coverage $(\%)$ & $53.8 \pm 13.6$ & $83.2 \pm 12.7$ & $83.4 \pm 7.6$ \\
\hline Posterior coverage (\%) & $51.9 \pm 14.9$ & $71.5 \pm 18.8$ & $80.1 \pm 7.8$ \\
\hline
\end{tabular}

Student's t test for paired data was used to determine statistical significance.

Significantly different from preoperation; ${ }^{*} \mathrm{p}<0.0001$
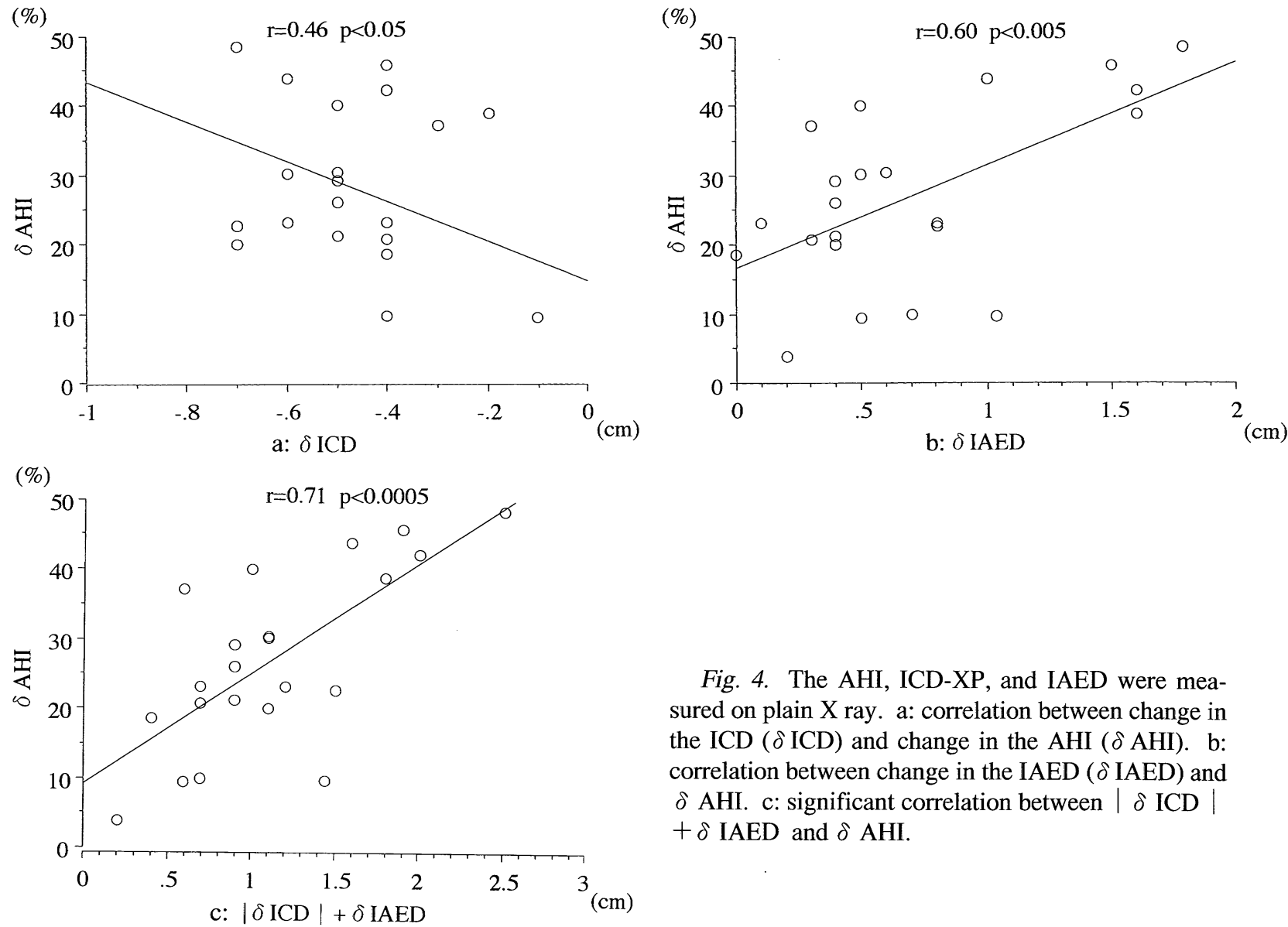

Fig. 4. The AHI, ICD-XP, and IAED were measured on plain $\mathrm{X}$ ray. a: correlation between change in the ICD ( $\delta$ ICD) and change in the AHI ( $\delta$ AHI). b: correlation between change in the IAED ( $\delta$ IAED) and $\delta$ AHI. c: significant correlation between $\mid \delta$ ICD $\mid$ $+\delta$ IAED and $\delta$ AHI. 
and also in the IAED, were correlated with changes in the AHI $(r=-0.46, p<0.05$, and $r=0.60, p<0.005$, respectively) (Figs $4 a, b$ ). Therefore the sum of the difference in the ICD and IAED was correlated with the absolute difference in the AHI $(r=0.71, p<0.0005)$ (Fig. 4c). This evidence suggested that displacements of the fragments after Chiari pelvic osteotomy occurred both proximally and distally.

Comparison of computer tomograms taken before and after surgery found that the ISD was significantly increased after surgery by an average of $0.8 \pm 0.4 \mathrm{~cm}(\mathrm{p}<0.0001)$, and that the IA was significantly decreased by an average of $2.7 \pm 2.5$ degrees $(\mathrm{p}<0.0001)$. These findings indicated lateralization of the proximal fragment. The ICD significantly decreased by an average of $0.4 \pm 0.2 \mathrm{~cm}$ $(p<0.0001)$, indicating medialization in the distal fragment. The AASA was not significantly changed (n.s.), the PASA was significantly decreased by an average of $2.7 \pm 5.1$ degrees $(p<0.05)$, and the AcAV was significantly decreased by an average of $1.6 \pm 2.5$ degrees $(\mathrm{p}<0.05)$. DBCS was not signifi- cantly changed (n.s.) (Table 2).

The anterior acetabular coverage over the femoral head improved by an average $29.4 \pm 10.8 \%$ of the anterior part of femoral head, while the posterior coverage improved by an average of $19.6 \pm 14.8 \%$ (Table 3).

\section{DISCUSSION}

Measurements of the CE angle and changes in the location of the center of the femoral head on plain X-ray have previously suggested that medialization of the center of the femoral head and lateralization in the distal fragment occurred following Chiari pelvic osteotomy, thus increasing the load area [15]. Similarly, in this study using plain Xray, changes in the ICD and IAED were correlated with the AHI. Changes in the IAED were correlated with the AHI more so than changes in the ICD. In addition, the sum of changes in the ICD and IAED were well correlated with the AHI. However, plain $\mathrm{X}$-ray analysis could not determine three-dimen-

TABLE 4.

Anatomical changes in the pelvis after surgery

\begin{tabular}{|c|c|c|c|c|c|c|c|c|}
\hline \multirow{2}{*}{ case } & \multirow{2}{*}{$\operatorname{sex}$} & \multirow{2}{*}{ age } & \multirow{2}{*}{ stage } & \multirow{2}{*}{ operation } & \multirow{2}{*}{$\begin{array}{l}\text { proximal fragment } \\
\text { lateralization }\end{array}$} & \multicolumn{3}{|c|}{ distal fragment } \\
\hline & & & & & & medialization & rotation & a-p displacement \\
\hline 1. T. I & f. & 41 & III & $\mathrm{C}+\mathrm{v}$ & large & medium & anterior & anterior \\
\hline 2. H. I & f. & 44 & III & $\mathrm{C}$ & large & medium & anterior & - \\
\hline 3. H. U & f. & 49 & II & $\mathrm{C}$ & large & large & - & - \\
\hline 4. N. E & f. & 41 & III & $\mathrm{C}$ & small & medium & - & - \\
\hline 5. R. O & f. & 40 & IV & $\mathrm{C}+\mathrm{v}$ & small & small & anterior & anterior \\
\hline 6. Y. O & f. & 48 & III & $\mathrm{C}+\mathrm{v}$ & medium & small & posterior & posterior \\
\hline 7. S. K & f. & 40 & II & $C+v$ & large & medium & anterior & - \\
\hline 8. S. K & f. & 21 & II & $\mathrm{C}+\mathrm{v}$ & medium & medium & anterior & - \\
\hline 9. S. K & f. & 35 & III & $\mathrm{C}+\mathrm{v}$ & small & medium & anterior & - \\
\hline 10. K. S & f. & 52 & III & $\mathrm{C}+\mathrm{v}$ & medium & small & anterior & posterior \\
\hline 11. E. T & f. & 52 & III & $\mathrm{C}+\mathrm{v}$ & large & medium & - & - \\
\hline 12. K. T & f. & 29 & I & $\mathrm{C}$ & large & medium & anterior & - \\
\hline 13. R. N & f. & 39 & II & $\mathrm{C}$ & medium & large & - & - \\
\hline 14. Y. N & f. & 41 & III & $C+v$ & medium & medium & posterior & - \\
\hline 15. M. N & f. & 39 & II & $\mathrm{C}$ & medium & small & - & - \\
\hline 16. M. H & f. & 45 & IV & $C+v$ & large & small & anterior & anterior \\
\hline 17. A. $\mathrm{H}$ & f. & 55 & III & $C+v$ & large & small & posterior & - \\
\hline 18. E. F & f. & 36 & III & $\mathrm{C}+\mathrm{v}$ & medium & large & anterior & posterior \\
\hline 19. K. M & f. & 53 & III & $\mathrm{C}$ & large & medium & posterior & - \\
\hline 20. Y. Y & f. & 51 & III & $C+v$ & medium & small & anterior & - \\
\hline 21. T. Y & $\mathrm{m}$. & 23 & II & $\mathrm{C}$ & large & large & - & - \\
\hline 22. H. Y & f. & 57 & II & $\mathrm{C}+\mathrm{v}$ & medium & small & anterior & posterior \\
\hline
\end{tabular}

$\mathrm{C}$ : Chiari pelvic osteotomy; $\mathrm{C}+\mathrm{v}$ : Chiari pelvic osteotomy and simultaneous valgus osteotomy of the fumer small $\leqq 0.3 \mathrm{~cm}, 0.3 \mathrm{~cm}<$ medium $\leqq 0.6 \mathrm{~cm}, 0.6 \mathrm{~cm}<$ large; - : changeless 


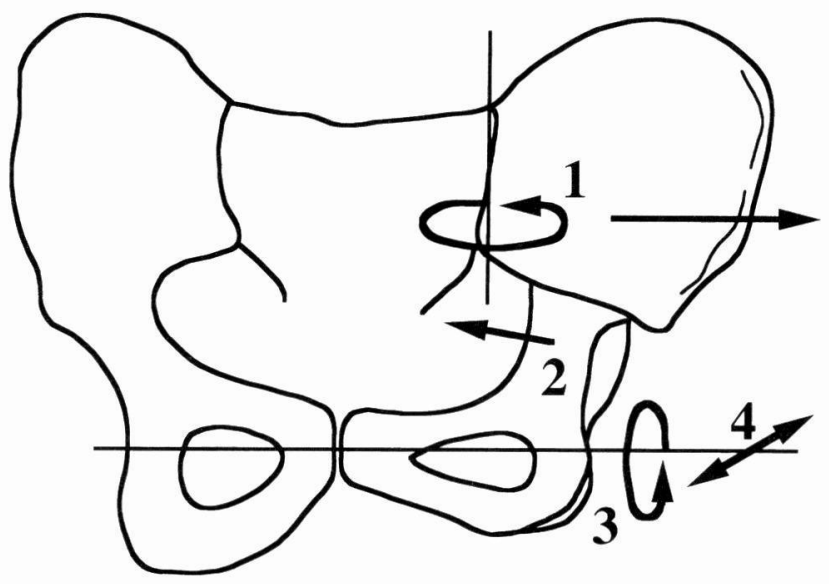

Fig. 5. 1: Lateral displacement in the proximal fragment occurred around the axis of the sacroiliac joint. 2: Medial displacement in the distal fragment occurred. 3: Anterior rotation in the distal fragment occurred. 4: In anterior-posterior direction, various displacement in the distal fragment occurred.

sional movements in the fragments. This CT study confirmed that anatomical changes in the pelvis following Chiari pelvic osteotomy involved significant increases in the postoperative ISD, which were consistent with the findings (namely an increase in the IAED) obtained by plain anterior and posterior X-ray analysis. I was also able to capture significant decreases in the IA at the level of the anterior superior iliac spine as actual angles, which indicated lateralization in the proximal fragment as a widening movement around the axis of the sacroiliac joint. In addition, the ICD at the level of the center of the femoral head was decreased, indicating medialization in the distal fragment.

In order to observe the movements in the distal fragment in the sagittal plane, the inclination of the pelvis was investigated in terms of the AASA, PASA and AcAV as defined by Anda et al. [12,13]. When the pelvis was tilted anteriorly, then the AcAV and PASA were each decreased, while the AASA was increased.

The AcAV and PASA were each significantly decreased after surgery. In other words, the distal fragment was rotated anteriorly around the axis of the pubic symphysis. Furthermore, the DBCS was variously changed. In other words, the distal fragment was variously displaced anteriorly, posteriorly, or neither (Fig. 5, Table 4). It was suggested that a different curvature of the dome by dome osteotomy, a different stiffness in the pubic symphysis were two of the reasons for this variation in the DBCS.

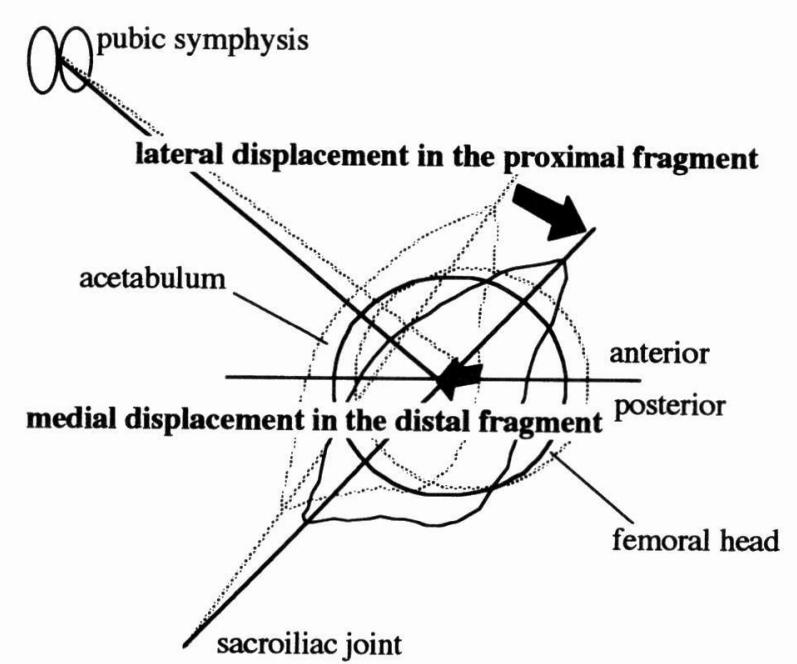

Fig. 6. In addition to medial displacement in the distal fragment, lateral displacement in the proximal fragment was also related to anterior coverage.

The acetabular coverage over the femoral head was significantly increased in the anterior half and in the posterior half after surgery, with a greater increase seen in the anterior half. This was consistent with Klaue et al.'s report [14] that the femoral head coverage was poorer in the posterior part. These findings suggested that medialization in the distal fragment and lateralization in the proximal fragment around an axis of the sacroiliac joint were associated with improvement in the anterior acetabular coverage over the femoral head (Fig. 6). This was consistent with the medialization in the distal fragment (change in the ICD-XP, CT) which was less than the lateral displacement in the proximal fragment (change in the IAED and ISD). Medialization occurred in the distal fragment in the 14 cases and to a slight extent $(\leqq 0.3$ $\mathrm{mm}$ ) in the other 8 cases. On the other hand, lateralization in the proximal fragment occurred in 19 cases and to a slight extent $(\leqq 0.3 \mathrm{~mm})$ in the other 3 cases (Table 4). Chiari consisted that medialization in the distal fragment was related to the acetabular coverage over the femoral head. However in the present study, it was suggested that lateralization in the proximal fragment was correlated more closely with the acetabular coverage over the femoral head coverage, than medialization in the distal fragment. Furthermore, it was suggested that the muscle release effect was brought about through the lateralization in the proximal fragment.

These tendencies were observed, through to various extents due to a different stiffness in the 
pubic symphysis and sacroiliac joint, or other reason. This study was only concerned with unilateral surgery, and different factors may be involved in bilateral surgery.

\section{CONCLUSIONS}

This study revealed anatomical changes in the pelvis after modified Chiari pelvic osteotomy using CT. It was suggested that the acetabular coverage over the femoral head was more affected by lateralization in the proximal fragment than medialization in the distal fragment. In the sagittal plane, anterior rotation in the distal fragment tended to be occurred, while the a-p displacement was various.

ACKNOWLEDGMENTS: The author thanks Prof. A. Inoue and Associate Prof. F. Higuchi (Department of Orthopaedic Surgery, Kurume University School of Medicine) for clinical advice and suggestions.

\section{REFERENCES}

1. Chiari K. Beckenostenotomie zur Pfannendachplastik. Wien Med Wochenschr 1953; 103:707-714.

2. Chiari K. Medial displacement osteotomy of the pelvis. Clin Orthop 1974; 98:55-72.

3. Küsswetter W, and Magers H. Changes in the pelvis after the Chiari and Salter osteotomy. Int Orthop (SICOT) 1985; 9:139-146.

4. Betz RR, Kumar SJ, Palmer CT, and MacEwen GD. Chiari pelvic osteotomy in children and young adults. J Bone Joint Surg (Am) 1988; 70-A:182-191.

5. Fujii G, Sakurai M, Kumeta $H$, and Funayama K.
Biomechanical analysis after Chiari's pelvic osteotomy by means of superimposed pelvic computed tomography scan. In: Hip Biomechanics, Imura S et al., SpringerVerlag, Berlin, pp 205-213, 1993.

6. Vigliani F, Bonaga S, and Marin G. Preoperative and postoperative evaluations by means of three-dimensional computed tomography in cases of Chiari osteotomy. Clin Orthop 1991; 266:104-110.

7. Kawamura B. The transverse pelvic osteotomy. J Jap Orthop Surg Soc 1959; 32:65-72.

8. Inoue A, Higuchi F, and Shiba N. Chiari pelvic osteotomy for coxarthrosis in adults. J Orthop Surg Tech 1990; 5:105-111.

9. Matsuno T, Ichioka Y, and Kaneda K. Modified Chiari pelvic osteotomy: a long term follow-up study. J Bone Joint Surg (Am) 1972; 74-A:470-478.

10. Anwer MM, Sugano M, Matsui M, Takaoka K, and Ono $\mathrm{K}$. Dome osteotomy of the pelvis for osteoarthritis secondary to hip dysplasia: A five-year follow-up study. J Bone Joint Surg (Br) 1993; 75-B:222-227.

11. Heyman $\mathrm{CH}$, and Herdon $\mathrm{CH}$. Legg-Perthes disease: a method of the measurement of the rentogenographic result. J Bone Joint Surg (Am) 1950; 32-A:767-768.

12. Anda S, Svenningsen S, Dale LG, and Benum P. The acetabular sector angle of the adult hip determined by computed tomography. Acta Radiol 1986; 27:443-447.

13. Anda S, Svenningsen S, Grontvet T, and Benum P. Pelvic inclination and spatial orientation of the acetabulum. A radiographic, computed tomographic and clinical investigation. Acta Radiol 1990; 31:389-394.

14. Kraue K, Sherman M, Perren SM, Wallin A, Looser C et al. Extra-articular augmentation for residual hip dysplasia. J Bone Joint Surg (Br) 1993; 75-B:750-754.

15. Antolic V, Srakar F, Kralj-Iglic V, Iglic A, ZaletelKragelj L et al. Changes in configuration of the hip due to Chiari osteotomy. Orthop 1996; 4:183-186. 